\title{
Applications of Dynamic Mechanical Analysis in the Engineering of Amorphous Solid Dispersions
}

\author{
Andrew Toye Ojo ${ }^{1}$ Ping I. Lee ${ }^{1}$ \\ ${ }^{1}$ Department of Pharmaceutical Sciences, Leslie Dan Faculty of \\ Pharmacy, University of Toronto, Toronto, Ontario, Canada \\ Pharmaceut Fronts 2020;2:e55-e63.
}

\begin{abstract}
Address for correspondence Ping I. Lee, PhD, Leslie Dan Faculty of Pharmacy, University of Toronto, 144 College Street, Toronto, Ontario M5S 3M2, Canada (e-mail: ping.lee@utoronto.ca).
\end{abstract}

\author{
Abstract \\ Keywords \\ - Amorphous solid \\ dispersions \\ - dynamic mechanical \\ analysis \\ - hot melt extrusion \\ - pharmaceutical \\ - physical stability
}

Dynamic mechanical analysis (DMA) offers several advantages over prevailing methods in the characterization of amorphous solid dispersions (ASDs) typically used for improving the delivery of poorly water-soluble drugs. This method of analysis, though underutilized in the study of pharmaceutical systems, is particularly attuned to rheological investigations of thermal and mechanical properties of solids such as ASDs. Its ability to determine the viscoelastic properties of systems across a wide range of temperatures and shear conditions provides useful insight for the development and processing of ASDs. The response of materials to an imposed stress, captured by DMA, can help identify proper conditions for preparing homogenous extrudates of the polymer and active pharmaceutical ingredient through hot melt extrusion (HME). As HME continues to gain utility within the pharmaceutical industry, the ability to tailor process conditions will become increasingly important for the efficient design and production of ASD products for poorly water-soluble drugs. Furthermore, DMA can be used to probe molecular mobility and its link to physical stability of ASDs. Establishing the link between molecular mobility and crystallization kinetics is central to predicting the physical stability of ASDs. Therefore, increasing the understanding of material properties through DMA will enable the successful development of more stable amorphous drug products. This review summarizes current characterization tools for ASDs and discusses the potential of utilizing DMA as a robust alternative to traditional methods.

\section{Introduction}

The formation of amorphous solid dispersions (ASDs) of a poorly soluble drug in suitable polymer carriers is an innovative and enabling technique for enhancing the dissolution and oral delivery of many poorly water-soluble active pharmaceutical ingredients (APIs). ${ }^{1}$ The approach has become increasingly important due to the growing number of poorly water-soluble APIs identified in the drug discovery process. ${ }^{2,3}$ These ASD systems can achieve a transiently higher solubility and a faster dissolution rate due to the inherently more energetic amorphous form of a poorly soluble API as compared with that of the pure crystalline drug, thereby increasing the diffusional driving force to enable enhanced drug absorption with improved bioavailability. ${ }^{4-7}$ The dissolution of ASDs and the evolution of the resulting supersaturation profiles and their in vivo implications have been the subject of many recent investigations. ${ }^{8-12}$ Instead, this review will focus on the application of dynamic mechanical analysis (DMA) to elucidate the solid-state stability and hot-melt processing properties of ASDs.

Solvent evaporation and thermal processing are two primary methods employed to manufacture ASDs at a large scale in the pharmaceutical industry. The application of the solvent method, principally through spray drying, has been limited due to the high costs associated with handling organic solvents and their recovery to mitigate the potential toxicity from solvent exposure. ${ }^{13}$ On the other hand, thermal preparation of ASDs can be achieved by the hot melt extrusion (HME) 
process, which involves melt-blending and extrusion of an API mixed with a suitable polymer excipient. The HME process has quickly become a prevailing method for the preparation of ASDs due to interests in more efficient continuous manufacturing and its ease of scaling up. ${ }^{14}$ DMA can be applied to the characterization of ASDs produced by either process, but is also particularly useful for the proper selection of HMEprocessing conditions.

DMA is an important testing technique that characterizes the thermal and mechanical properties of solid materials and polymer melts as a function of time, temperature, frequency, strain, and stress. ${ }^{6}$ During testing, stress or strain can be applied to a sample to probe its response to deformation and determine relevant material properties, such as viscosity, which are critical to the development of ASDs. Typically, a DMA instrument applies an oscillating force (stress) and records an oscillating sample response. Here, modulus is calculated from the elastic response, and damping is calculated from the viscous response. DMA measurements can be acquired across a wide range of conditions including those extremes inaccessible to traditional rheology due to instrument torque limitations. A detailed comparison with other characterization techniques will be presented later in this article.

Due to the analogous relationship between shear and oscillatory deformation, DMA can be a useful tool to determine HME-processing conditions. The shear thinning behavior of ASDs and the increase in flowability with temperature can be captured with DMA and used to delineate the processing window for HME. This is particularly important when APIs are thermally ${ }^{3,15}$ unstable or prone to shear degradation. ${ }^{15}$

The physical stability of ASDs, with respect to API recrystallization, has restricted their widespread use and adoption. In ASD systems with drug loading levels in excess of their thermodynamic solubility, physical stability defines their shelf-life and potential to be introduced as a drug product. Molecular mobility contributes significantly to the physical stability of ASDs ${ }^{16,17}$ and is therefore important to characterize to gain insight as to whether an amorphous API has been effectively stabilized in the polymer carrier. DMA can be applied to evaluate the molecular dynamics in ASDs through measurements of properties such as viscosity (related to diffusivity) and glass transition temperature $\left(T_{\mathrm{g}}\right)$, furthering our understanding on the ASD physical stability. Additionally, understanding physical properties that affect drug release from ASDs is also important when examining the dissolution behavior of drug-polymer composites.

Understandably, optimizing processing conditions, performance evaluations, and the assessment of stability are essential endeavors that support the successful development of ASDs. The objective of this review is to provide an overview of the DMA as a robust tool in the characterization and production of ASDs. DMA will also be compared with prevailing analytical methods.

\section{Materials and Methods}

\section{Materials}

Naproxen $\left(\mathrm{C}_{14} \mathrm{H}_{14} \mathrm{O}_{3}\right)$ was purchased from API Vanguard (United States) and used as received. Indomethacin $\left(\mathrm{C}_{19} \mathrm{H}_{16} \mathrm{ClNO}_{4}\right)$ and ketoconazole $\left(\mathrm{C}_{15} \mathrm{H}_{12} \mathrm{~N}_{2} \mathrm{O}\right)$ were purchased from Shanghai Yuxi Biotech Co., Ltd. (Shanghai, China) and used without further purification. Polyvinyl acetate $(500,000 \mathrm{~g} / \mathrm{mol})$ was purchased from Sigma-Aldrich Canada. HPMC HME 4M (AFFINISOL) was generously provided by Dow Chemical. All other chemicals and solvents were reagent grade and obtained commercially. The chemical structures of the APIs and the polymers are shown in -Fig. 1.

\section{Experimental Methods}

DMA was conducted on a TA Q800 instrument. Samples were prepared by melt compression to form rectangular-shaped specimens. Oscillatory rheology was performed to obtain the

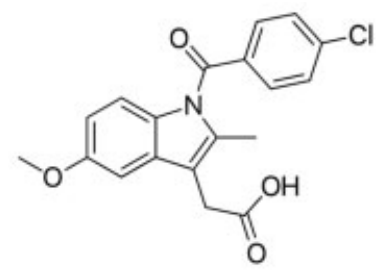

Indomethacin (a)<smiles>COc1ccc2cc([C@@H](C)C(=O)O)ccc2c1</smiles>

Naproxen (b)<smiles>CC(=O)OC(CC(C)(C)C)C(C)(C)C</smiles>

PVAc (c)

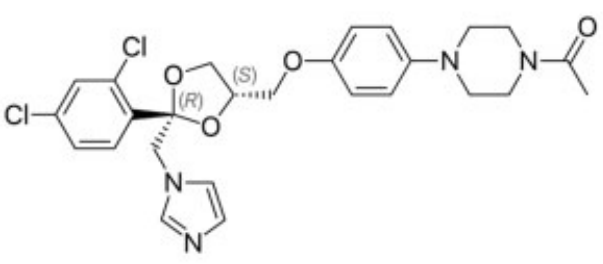

Ketoconazole (d)

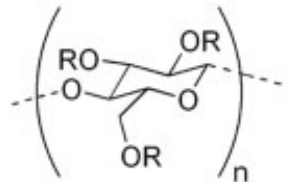

$\mathrm{R}=\mathrm{H}$ or $\mathrm{CH}_{3}$ or $\mathrm{CH}_{2} \mathrm{CH}(\mathrm{OH}) \mathrm{CH}_{3}$

HPMC 4M (e)

Fig. 1 Chemical structures of indomethacin (a), naproxen (b), PVAc (c), ketoconazole (d), and HPMC 4M (e). 
viscosity of model ASDs based on PVAc and HPMC. Strain was set to $0.02 \%$ for oscillations between 100 and $0.05 \mathrm{~Hz}$, with five points collected per decade. The Cross model was used to fit the frequency sweep data at multiple temperatures to determine zero-frequency viscosity. The Cross model, commonly applied in rheology, describes the shear-thinning behavior of polymer systems. ${ }^{18}$ Low-frequency data were used as an analog for the low shear data according to the CoxMerz rule to obtain the unperturbed zero-shear viscosity. ${ }^{19}$

Methods of ASD Characterization (Molecular Mobility) Several methods and experimental techniques are typically employed for the characterization of ASDs. Each provides critical information regarding the physical and chemical nature of such systems. Combining insights from multiple methods can contribute to a holistic understanding of ASD systems. The utility of methods aimed at probing the molecular mobility in ASDs will be discussed here.

Fourier-transform infrared spectroscopy (FTIR) and Raman spectroscopy: Microscopy is commonly employed to investigate polymorphism and identify crystallization. However, its application can also be extended to diffusive measurements. Raman has been used to probe diffusivity in ASDs by looking at the migration of molecules across an interface into a blank film. ${ }^{20}$ ATR-FTIR has also been used to probe the diffusion of molecular species in polymer systems. ${ }^{21}$ These methods rely on the characteristic signals or absorbance of molecules in response to excitation. The intensity of identifying peaks or absorption spectra can be used to determine relative concentration ratios of components and spatial concentration distributions for diffusion modeling purposes. However, the sensitivity and accuracy of these spectroscopic approaches can be impacted by chemical interactions leading to peak shifts. Additionally, the methods are limited to elevated temperature ranges required to achieve significant and measurable diffusion in a reasonable time frame.

Fluorescence spectroscopy: The diffusion of small molecules within polymer systems can be measured precisely with florescence-based techniques. Forced Rayleigh scattering and fluorescence recovery after photobleaching are two florescence-based approaches that are especially useful for the measurement of slow diffusion ${ }^{22}$ with diffusion coefficients on the order of $10^{-19} \mathrm{~m}^{2} / \mathrm{s}$. The decay of the photochemical response of fluorescent molecules allows relaxation times that are associated with the diffusion coefficients of "dye" molecules to be determined. The sensitivity in measuring photochemical processes involving molecular motion allows diffusion to be probed at both pharmaceutically relevant storage temperatures and elevated temperatures. However, measurements are limited to fluorophores that can be detected optically.

Mass spectrometry: Mass spectrometry is a highly sensitive analytical technique that can identify and quantify molecules in complex systems. Coupled with depth profiling or desorption, molecular distributions can be determined on a nanometer scale and used to elucidate diffusion coefficients. ${ }^{23,24}$ Disadvantages of probing molecular mobility with mass spectrometry include the difficulty of method development, high instrument cost, and a limited scope of experiments requiring multiple experiments to garner information over a broad range of temperatures or conditions.

Molecular relaxation (nuclear magnetic resonance [NMR] and dielectric spectroscopy): The roles of polymer concentration on the molecular mobility and physical stability of nifedipine solid dispersions were recently investigated by Suryanarayanan and coworkers ${ }^{25}$ with the use of dielectric spectroscopy. The technique applies an alternating electrical field to investigate the movement of dipoles. A decrease in molecular mobility was observed with an increase in polymer concentration from 2.5 to $20 \%$. This method as well as others based on NMR relaxation failed to identify the polymer concentration needed to stabilize drugs in solid dispersions at room temperature and over a typical pharmaceutical shelf-life period. ${ }^{25-27}$ This can be attributed to the investigation of molecular mobility through material relaxation processes. As relaxation processes get slower, and the time scale of such experiments increases and eventually becomes impractically long for probing diffusion in ASDs.

Dissolution: Dissolution and sorption experiments can be conducted to assess API mobility in polymer carriers. ${ }^{28,29}$ These methods also allow both the diffusion and partition coefficients of an API in a polymer matrix to be determined. This is achieved by monitoring time-dependent changes in drug solution concentrations, determined spectroscopically, to elucidate diffusivity within a carrier. Approximate analytical solutions for drug absorption or drug release in a finite volume allow for the practical implementation of these methods. However, the hydration of a carrier matrix can impact the translation of measured drug diffusion coefficients to the dry and rigid solid state of ASDs. The higher drug diffusivity obtained due to polymer hydration and swelling will result in a considerable underestimation of the stability duration of ASDs. Additionally, hydration and swelling of ASDs can lead to phase separation and drug crystallization which, in turn, can impact transport processes within ASDs and shorten the stability duration.

Rheology: Rheology can be used to connect the physical properties of ASDs to their stability ${ }^{20}$ and processability. ${ }^{30}$ Although underutilized, it presents several advantages compared with the methods described above. By probing diffusion in the absence of solvents, solid-state characteristics of ASDs can be probed more accurately than dissolution-based approaches. Method development and data acquisition are also more straightforward than mass and florescence spectrometry. Additionally, probe molecules do not need to be fluorophores or radio-labeled. Peak shifts, due to drug-polymer interactions, that can hamper FTIR and Raman spectroscopy approaches, are also avoided. DMA offers considerable flexibility in terms of controlling humidity, temperature, and frequency conditions. Due to the higher measurement range than tradition rheology, DMA is better suited for the study of ASDs (- Fig. 2). In this case, low-frequency measurements are relevant to the stability of ASDs, whereas higher frequency measurements provide direct insight for the HME processing. Low-frequency oscillation data are as analogous to the lowshear data according to the Cox-Merz rule and can be used to 


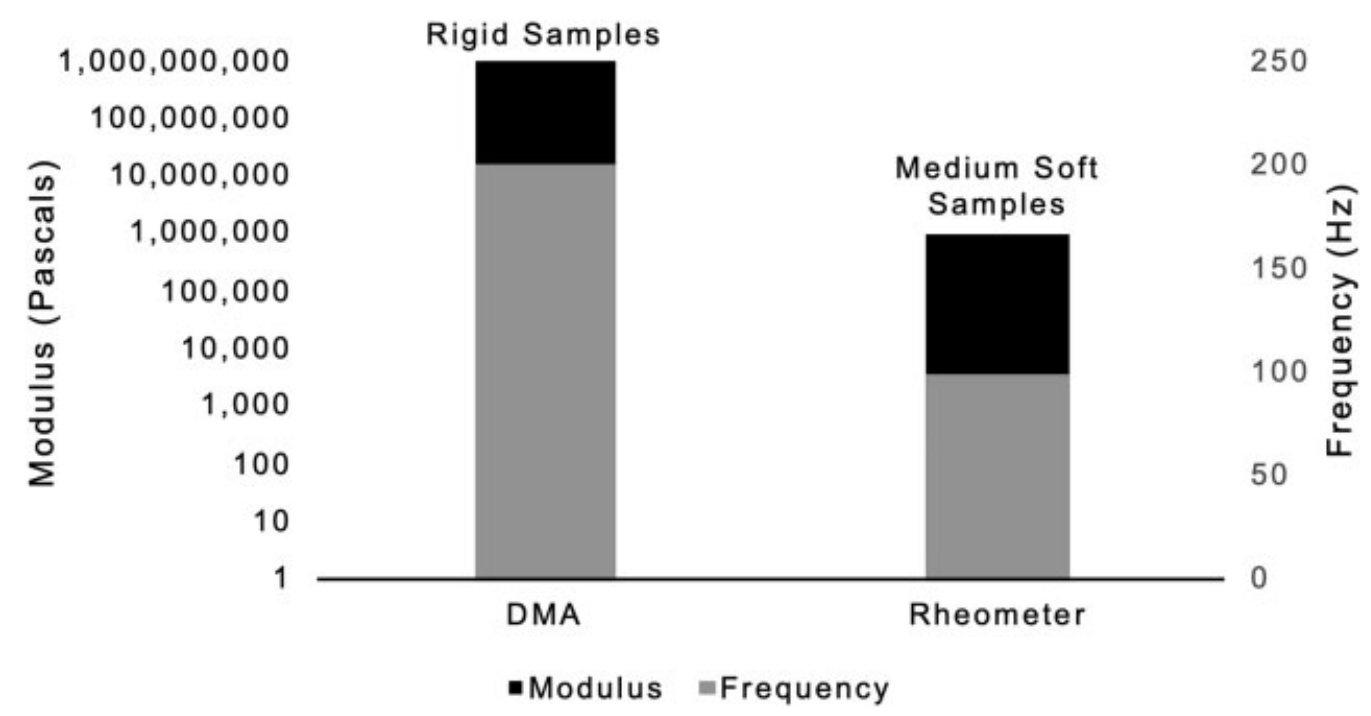

Fig. 2 Measurement range for DMA and conventional rheometers. DMA, dynamic mechanical analysis.

obtain the unperturbed zero-shear viscosity of ASDs. API diffusivity can then be determined from zero-shear viscosity and used in the current stability modeling of ASDs. A major drawback of the rheological approaches is that diffusivity may decouple from the viscosity and exhibit non-Arrhenius behavior below $1.2 \times T_{\mathrm{g}}$ and therefore must be accounted for by a fractional exponent relationship with viscosity. ${ }^{31}$ In pharmaceutical systems, the crystal growth rate can be determined from expressions that include this decoupling. ${ }^{32}$

\section{DMA as a Tool for Process Development}

HME has developed into a pivotal technology for the preparation of ASDs. As such, advances in process development have a direct impact on the creation of new ASD drug delivery systems. Melt rheology is an efficient tool ${ }^{30}$ which provides insight into material properties that are important for the HME-manufacturing process and can provide guidance for the process design. ${ }^{33}$ Temperature and screw speed are among the most important factors affecting both the HME processing and final ASD products through the distributive mixing and solubilization of APIs. ${ }^{34}$

Screening of various compositions can be performed effectively with the rheological study of drug: polymer physical mixtures (PMs). ${ }^{30}$ The softening temperatures of the PMs and viscosity at various shear rates can help guide the initial selection of composition, temperature, and shear conditions to carry out $\mathrm{HME}^{30}$ The smaller rheological sample size, compared with extrusion, can greatly reduce cost and expedite process development. The miscibility of components and their suitability for further product development as ASDs can be established by the appearance of a single glass transition temperature upon thermal analysis, whereas a multiphase mixture will result in multiple glass transition temperatures. DMA is typically 10 to 100 times more sensitive to changes occurring at $T_{\mathrm{g}}$ than DSC, ${ }^{35}$ allowing a better discrimination between amorphous solid solutions with molecular level dispersion and multiphase systems. ${ }^{30}$

During HME, increasing temperature reduces the melt viscosity of polymers, thereby increasing the processability.
Typically, extrusion temperatures must be at least 10 to $20^{\circ} \mathrm{C}$ higher than the glass transition temperature of the polymer being used..$^{36}$ Otherwise, the torque or load on an extruder's motor would be too high to rotate. ${ }^{37}$ The relationship between temperature and melt viscosity is Arrhenius in nature. ${ }^{37}$

$$
\eta=K^{\prime} \cdot e^{\frac{E_{\mathrm{a}}}{R T}}
$$

where, $K^{\prime}$ is a constant, $\eta$ is the viscosity, $E_{\mathrm{a}}$ is the activation energy of the polymer flow process, $R$ is the gas constant, and $T$ is the temperature in kelvin.

Similarly, increasing shear reduces the melt viscosity of polymers due to their pseudoplastic shear-thinning behavior. The relationship between the shear rate and melt viscosity follows a power-law equation ${ }^{37}$ :

$$
\eta=K \cdot \gamma^{n-1}
$$

where $\gamma$ is the shear rate, $K$ is an exponential function of the temperature and polymer properties, and $n$ is the power-law constant.

Thus, temperature ${ }^{37}$ and shear ${ }^{37,38}$ together define the processing window for HME. These parameters define regions of processing conditions capable of generating the optimal melt viscosity for HME, as shown in -Fig. 3. It is critical to ensure that the extrusion equipment selected to prepare the desired ASD system can meet these requirements. Thermal and shear degradation further narrows the range of acceptable extrusion conditions. The drug loading level is also an important consideration when selecting processing conditions. Varying the composition of an ASD can cause a shift in optimal processing conditions that have been previously identified. APIs can act as plasticizers in polymer systems, thus greatly reducing the input of mechanical energy needed to melt and process ASDs by HME. The influence of temperature and drug loading level on the zero-shear viscosity of ASDs is illustrated in - Fig. 4. Therefore, by developing a complete mapping of the effects of temperature, shear, and drug loading level on melt viscosity, the optimal conditions for HME can be identified. 


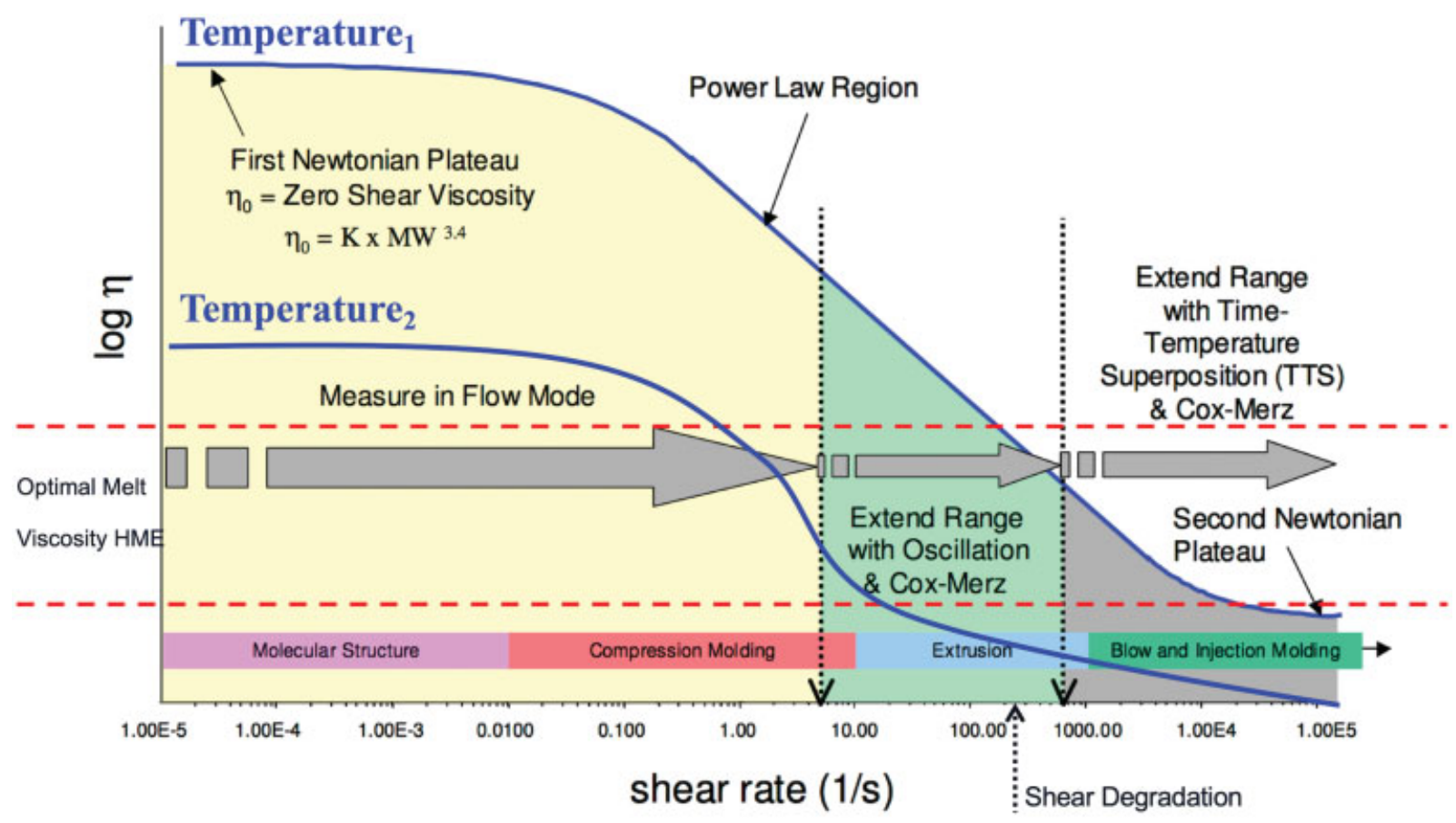

Fig. 3 Processing window to achieve the optimal melt viscosity for hot melt extrusion process as defined by temperature and shear [Adapted with permission from TA Instruments ${ }^{38}$.

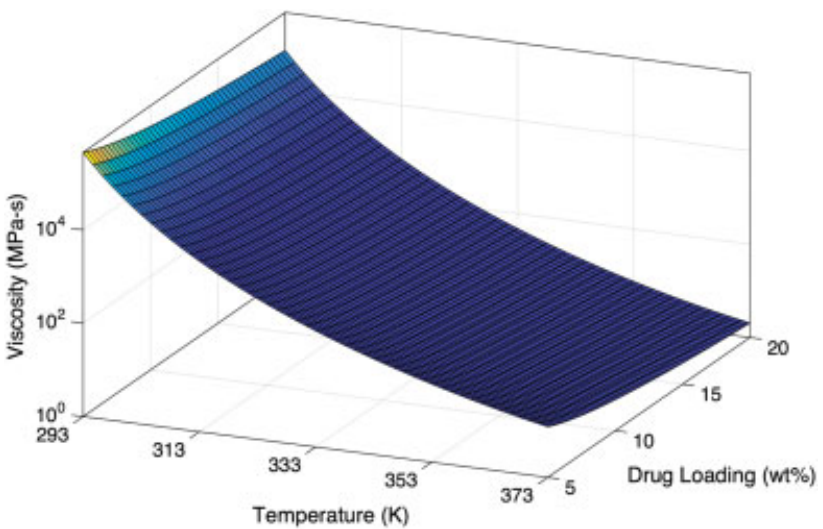

Fig. 4 Effect of temperature and drug loading level on the zero-shear viscosity of ASDs, determined by DMA (NAP:PVAc). ASDs were prepared with various compositions and subjected to temperature sweeps to generate the fitted surface plot of the data. The average of duplicate data was fitted. The Cross model was used for the zero-shear viscosity approximation from frequency data. ASD, amorphous solid dispersion; DMA, dynamic mechanical analysis.

\section{Material Characterization for ASD stability (Diffusivity/Stability, $T_{\mathbf{g}}$ )}

Although the use of ASDs has been shown to enhance the bioavailability of poorly water-soluble compounds, their development remains challenging. One major challenge arises from the difficulty in predicting ASD stability in the solid state. Earlier, we have shown the utility of DMA in determining the appropriate HME processing conditions for ASD preparation. Here, we will demonstrate how DMA can further our understanding of ASD properties and address stability questions that have remained unanswered.

The link between ASD stability and molecular mobility is well recognized. ${ }^{17,25,27}$ However, establishing a predictive

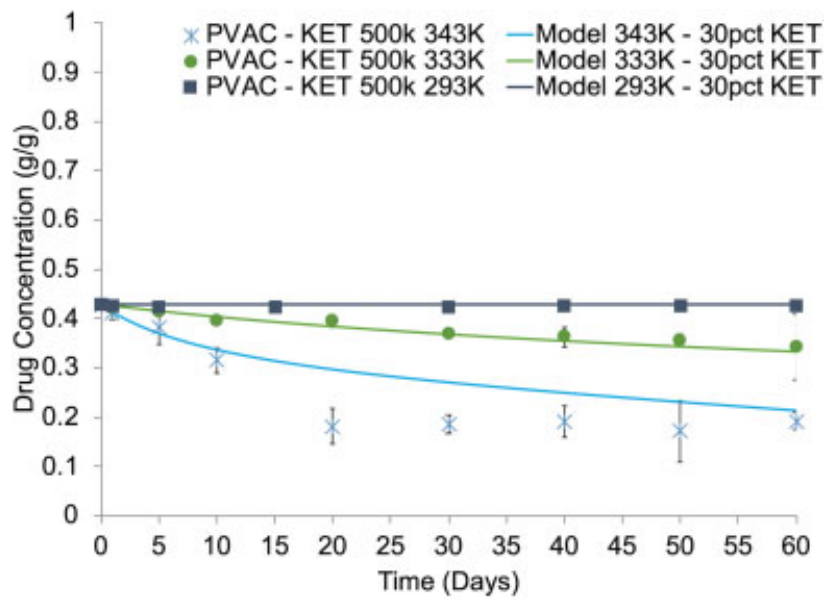

Fig. 5 Effect of temperature on the physical stability of KET:PVAC ASD systems with $30 \mathrm{wt} \%$ drug loading. Experimental data points are shown with lines representing trends predicted from model simulations. ASD, amorphous solid dispersion.

mathematical relationship between mobility and stability has been challenging due to the complexity of their coupling and the lack of a fundamental understanding of key parameters controlling ASD crystallization in the solid state, ${ }^{38}$ particularly the failure to consider supersaturation as the driving force for crystallization in the solid solution matrix of an ASD. Existing approaches are mostly empirical ${ }^{39-41}$ or correlative, ${ }^{42,43}$ considering parameters such as miscibility of drug in the polymer matrix or structure relaxation within the ASD, without much success in predicting the ASD stability . Recently, we have shown that a comprehensive model considering nucleation and growth processes can be used to describe the physical stability of ASDs in the solid state (see - Fig. 5). ${ }^{44}$ Central to our model was the determination 


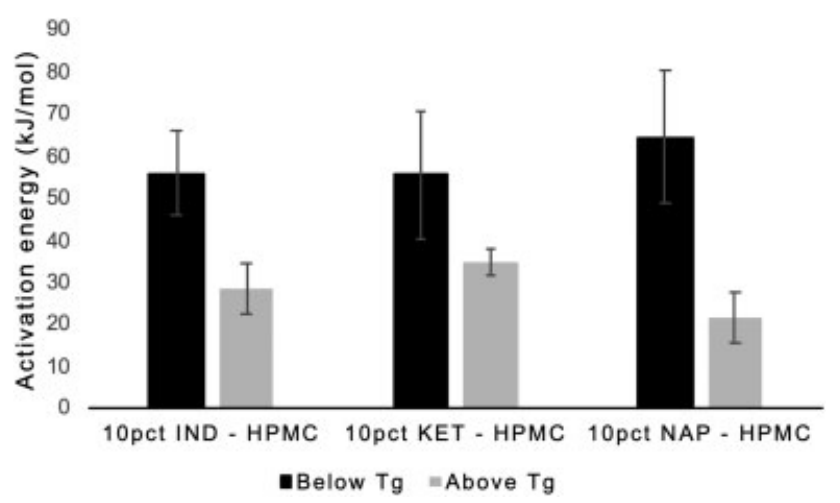

Fig. 6 Activation energy for API diffusion in HPMC at a $10 w t \%$ drug loading level, above and below $T_{\mathrm{g}}$. Error bars indicate standard deviations resulting from duplicate DMA measurements of viscosity. DMA, dynamic mechanical analysis.

of API diffusivity. This was done by exploring the relationship between viscosity and drug diffusivity. The activation energy for the diffusion process can then be determined from the temperature dependence of API mobility. - Fig. 6 and - Fig. 7 show the activation energies of diffusion measured for some of the model ASD systems.

At small undercoolings, the temperature dependence of molecular transport has been effectively correlated with the inverse of shear viscosity $\eta^{-1}$. However, the proportionality breaks down at a temperature near $T_{\mathrm{g}}$ and as undercooling increases (-Fig. 8). ${ }^{32}$ This phenomenon is of critical importance in glassy ASDs which are typically stored well below their $T_{\mathrm{g}}$. A mass spec methodology was developed by Ediger and coworkers to probe diffusion in these supercooled liquids and glasses. ${ }^{24}$ With this method, they quantified the weaker temperature dependence for self-diffusion than that of viscosity, as $\eta^{-0.8}$, at lower temperatures in supercooled o-terphenyl. Subsequent work showed that the weaker temperature dependence could be predicted precisely by a fragility-dependent decoupling coefficient (Eq. 3), and the process of crystal growth to be similar to that governing self-diffusion. ${ }^{32}$ Fragility is a key measure of glass-forming ability, which has been characterized for most APIs. ${ }^{45}$ It describes the tendency for APIs to undergo devitrification and correlates with physical stability of its amorphous form. The decoupling coefficient

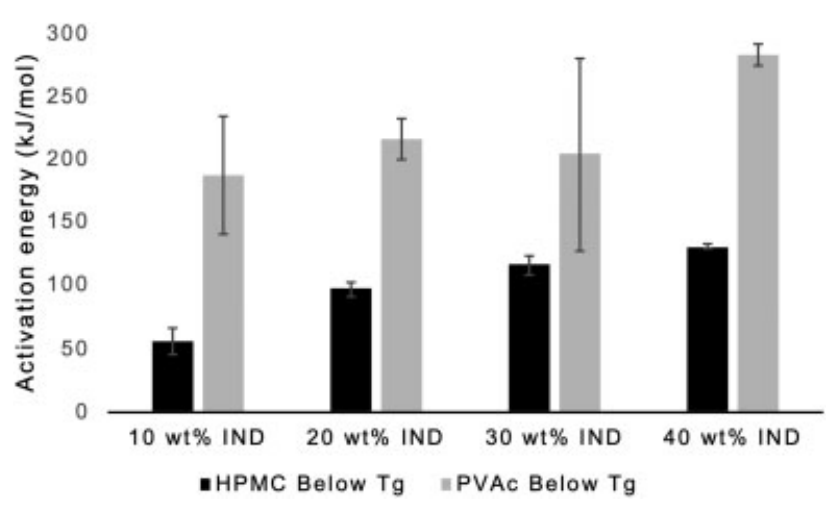

Fig. 7 Activation energy for IND diffusion in PVAC and HPMC, below $T_{\mathrm{g}}$. Error bars indicate standard deviations resulting from duplicate DMA measurements of viscosity. DMA, dynamic mechanical analysis.

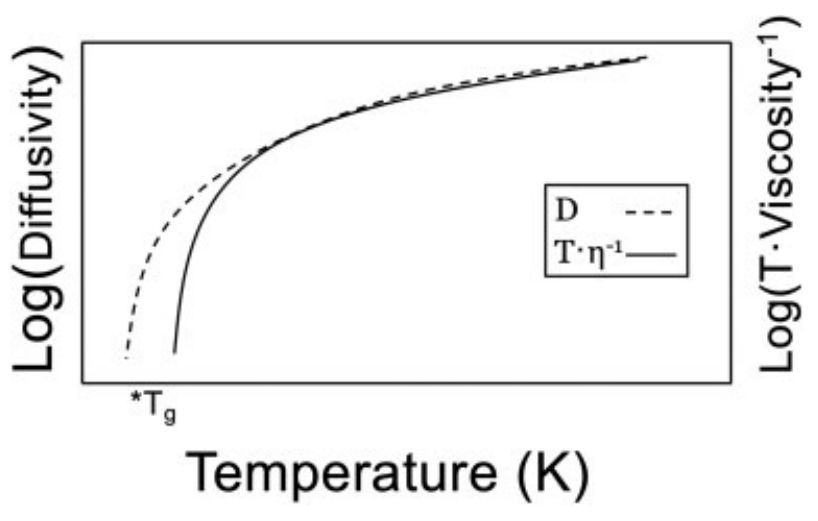

Fig. 8 Schematic representation of the decoupling between diffusion and viscosity near $T_{\mathrm{g}}$. Below $1.2 \times T_{\mathrm{g}}$, spatial heterogeneous dynamics enhance diffusion relative to the expected observations based on viscosity.

reflects a change in the underlying mechanism of motion in supercooled liquids and glasses. Spatial heterogeneity allows some molecules to find unhindered paths through materials allowing them to translate faster than normal. ${ }^{31}$ This leads to a microviscosity effect where the diffusion decouples from the viscosity $(\xi<1)$ and reflects the fact that the viscosity around a small molecule is rather different from that of the bulk due to the size effects. ${ }^{46}$ Viscosity decoupling and effective radius corrections $\left(R<R_{\mathrm{g}}\right)^{47}$ are methods that each addresses this discrepancy. In our work, we have used the more widely applied decoupling approach. Fractional equations can therefore be used to describe crystal growth and diffusion in supercooled liquids and glasses. Expressions for crystal growth (Eq. 4$)^{32}$ and self-diffusion (Eq. 5) ${ }^{48-50}$ have been validated with various experimental measurements at both elevated temperatures and near $T_{\mathrm{g}}$. In - Fig. 9, crystal growth rates, calculated from Eq. 4 over a range of temperatures, are compared for KET and IND ASDs in PVAc. It is therefore clear that DMA can be used to relate the bulk viscosity of ASDs to the stability-determining mobility of APIs within them.

Fragility-dependent decoupling:

$$
\epsilon=1.1-0.005 m
$$

where $m$ is fragility and $\epsilon$ the decoupling coefficient.

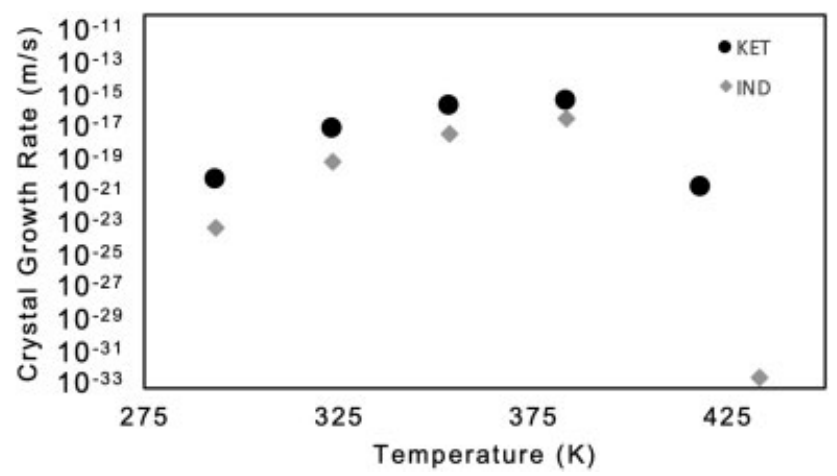

Fig. 9 Initial 2D surface-nucleated crystal growth rates $\left(r_{t}{ }^{0}\right)$ for KET and IND in PVAc ASDs at a drug loading level of $40 \mathrm{wt} \%$ at various temperatures determined with Eq. 4. ASD, amorphous solid dispersion. 
2D surface-nucleated crystal growth rate:

$$
r_{\mathrm{t}}(t)=\frac{\mathrm{d} r}{\mathrm{~d} t}=\frac{C T w}{\eta^{-\epsilon}}\left[1-\exp \left(-\frac{\Delta \mathrm{G}_{\mathrm{v}}}{k_{\mathrm{b}} T}\right)\right]
$$

where $C$ is a constant, $w$ reflects the growth mode, and $\Delta G_{\mathrm{v}}$ is the free energy change for crystallization, $T$ is temperature, and $k_{\mathrm{b}}$ is Boltzmann constant.

Fractional Stokes-Einstein diffusion equation:

$$
D=\frac{k_{\mathrm{b}} T}{6 \pi \eta^{-\epsilon_{R}}}
$$

where $D$ is diffusivity and $R$ is particle radius.

As established above, viscosity is central to developing the link between crystallization tendencies and molecular mobility. DMA allows the complex viscosity of ASDs to be measured across a wide range of temperatures to further our understanding on the effect of crystal growth rate on ASD stability. The response of polymer systems in such ASDs is viscoelastic, partially storing and dissipating energy upon shear. Complex viscosity accounts for these viscous and elastic contributions to the viscosity of the ASDs allowing a comprehensive relationship to be drawn between the microscopic motion of particles and the macroscopic viscoelastic response of a system. ${ }^{51}$ In the case of self-diffusion, this takes the form of the generalized Stokes-Einstein equation. In the case of a purely viscous fluid, the classical Stokes-Einstein equation would be recovered.

Generalized Stokes-Einstein diffusion equation:

$$
G^{*}(w)=\frac{k_{\mathrm{b}} T}{6 \pi R D^{*}(w)}
$$

where $G^{*}(w)$ is the complex shear modulus and $D^{*}$ is the diffusion coefficient.

Additionally, DMA is a useful tool for probing $T_{\mathrm{g}}$. Utilizing $T_{\mathrm{g}}$, on its own, is not a comprehensive approach for describing the physical stability of ASDs. However, identifying the temperature associated with transition from fluid to glassy dynamics is useful for identifying the temperatures at which the mobility in pharmaceutical glasses is strongly hindered thereby allowing the screening of drug-polymer combinations for their development potential. DMA is capable of characterizing this transition with exceptional precision. ${ }^{30}$

\section{Cox-Merz Rule and Cross Model}

Low-frequency data can be used as an analog for the low shear data according to the Cox-Merz rule for obtaining the unperturbed zero-shear viscosity for stability modelling. The CoxMerz rule is an empirical observation specifying that the steady-state zero-shear viscosity can be approximated from the analogous relationship between shear viscosity and oscillatory complex viscosity. ${ }^{19}$ These aspects of the Cox-Merz rule are illustrated in - Fig. 3, allowing DMA response curves to bound the processing conditions for ASDs and define their molecular mobility limiting zero-shear viscosity in the solid state. The Cross model can be used to fit the frequency sweep data ( - Fig. 10) to determine zero-shear viscosity. The model of Eq. 7, commonly employed in rheology, describes the shearthinning behavior of polymer systems enabling the determination of characteristic material properties. ${ }^{18}$

Cross model:

$$
\eta=\eta_{\infty}+\frac{\eta_{0}-\eta_{\infty}}{1+(c \gamma)^{m}}
$$

where $\eta_{0}$ is the zero-shear viscosity, $\eta_{\infty}$ is the infinite shear viscosity, $m$ is the Cross rate constant, and $C$ is the Cross time constant.

\section{Conclusion}

DMA is an established characterization tool that can be applied more broadly in the development of ASD systems.

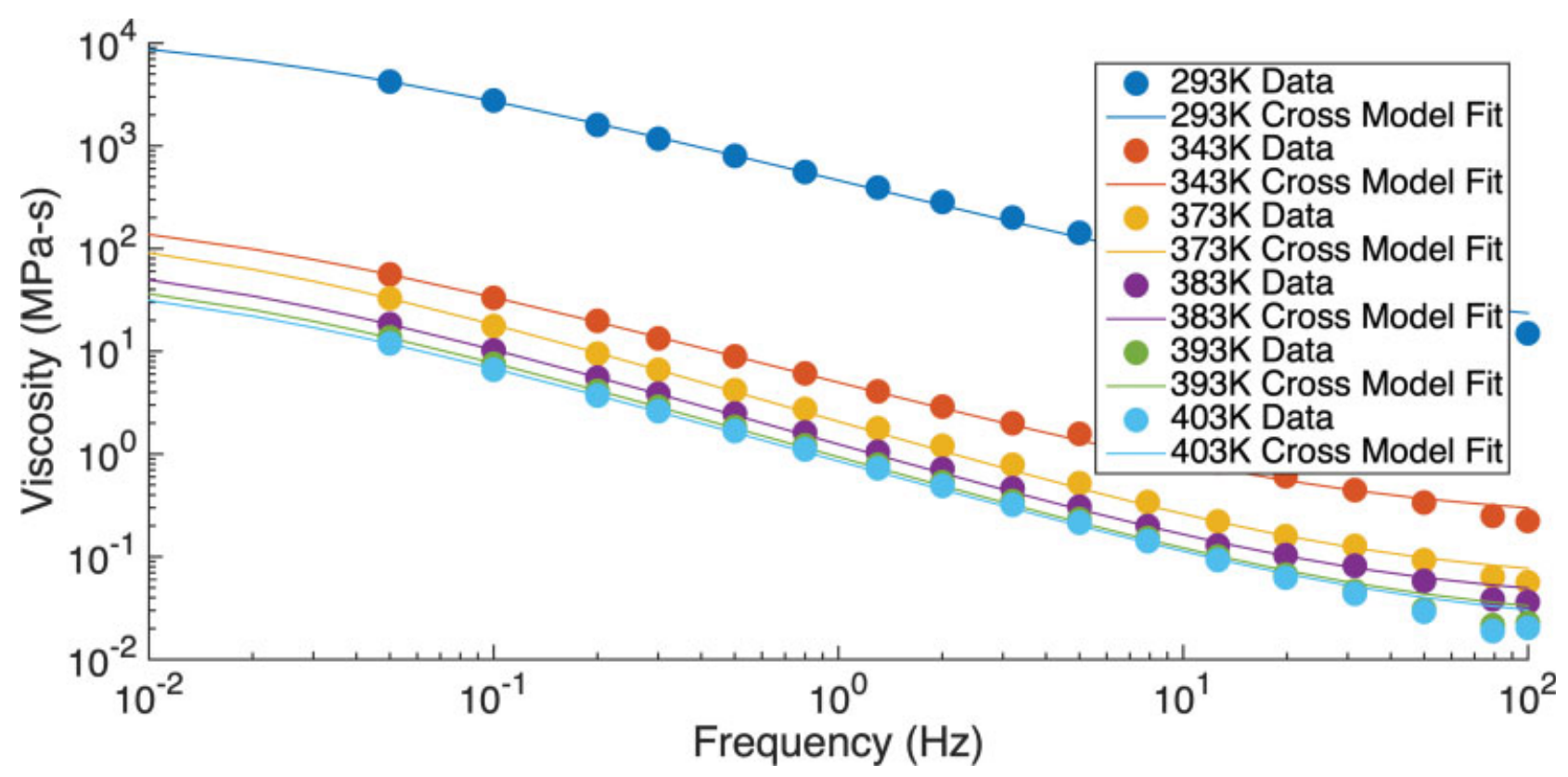

Fig. 10 Characteristic shear-thinning behavior of ASDs for oscillations between 100 and $0.05 \mathrm{~Hz}$, demonstrated with NAP:HPMC ASDs (30 wt\% drug loading) fitted with the Cross model. Viscosity is seen to decrease significantly with increasing temperature. ASD, amorphous solid dispersion. 
Characterization with DMA offers insights that are essential for both process development and stability modelling. As HME continues to emerge as a preeminent method of producing ASDs for the solubility enhancement of poorly soluble drugs, DMA can support the optimization of processing conditions. Stability modelling is also greatly facilitated by the broad range of thermomechanical insights provided by the DMA data that are relevant to phase changes occurring in ASD systems over time. Therefore, by relating critical properties of ASDs to manufacturing and formulation stability outcomes, the adoption and utilization of ASDs can be facilitated to enhance the delivery of poorly soluble drugs to better meet the needs of patients.

\section{Conflict of Interest}

The authors declare no competing financial interest.

\section{Acknowledgments}

This work was supported in part by funding from the Natural Sciences and Engineering Research Council of Canada (NSERC). A.T.O. was supported by a University of Toronto fellowship award. The authors would like to thank Rob Stehlik for the custom machining of DMA molds and extruder components.

\section{References}

1 Van Duong T, Van den Mooter G. The role of the carrier in the formulation of pharmaceutical solid dispersions. Part II: amorphous carriers. Expert Opin Drug Deliv 2016;13(12):1681-1694

2 Hitzer P, Bäuerle T, Drieschner T, et al. Process analytical techniques for hot-melt extrusion and their application to amorphous solid dispersions. Anal Bioanal Chem 2017;409(18):4321-4333

3 Liu J, Cao F, Zhang C, et al. Use of polymer combinations in the preparation of solid dispersions of a thermally unstable drug by hot-melt extrusion. Acta Pharm Sin B 2013;3(04):263-272

4 Goldberg AH, Gibaldi M, Kanig JL. Increasing dissolution rates and gastrointestinal absorption of drugs via solid solutions and eutectic mixtures III: experimental evaluation of griseofulvin-succinic acid solid solution. J Pharm Sci 1966;55(05):487-492

5 Simonelli AP, Mehta SC, Higuchi WI. Dissolution rates of high energy polyvinylpyrrolidone (PVP)-sulfathiazole coprecipitates. J Pharm Sci 1969;58(05):538-549

6 Chiou WL, Riegelman S. Preparation and dissolution characteristics of several fast-release solid dispersions of griseofulvin. J Pharm Sci 1969;58(12):1505-1510

7 Janssens S, Van den Mooter G. Review: physical chemistry of solid dispersions. J Pharm Pharmacol 2009;61(12):1571-1586

8 Sun DD, Lee PI. Evolution of supersaturation of amorphous pharmaceuticals: the effect of rate of supersaturation generation. Mol Pharm 2013;10(11):4330-4346

9 Augustijns P, Brewster ME. Supersaturating drug delivery systems: fast is not necessarily good enough. J Pharm Sci 2012;101(01):7-9

10 Sun DD, Lee PI. Haste makes waste: the interplay between dissolution and precipitation of supersaturating formulations. AAPS J 2015;17(06):1317-1326

11 Han YR, Lee PI. Effect of extent of supersaturation on the evolution of kinetic solubility profiles. Mol Pharm 2017;14(01):206-220

12 Sun DD, Lee PI. Probing the mechanisms of drug release from amorphous solid dispersions in medium-soluble and mediuminsoluble carriers. J Control Release 2015;211:85-93

13 Chiou WL, Riegelman S. Pharmaceutical applications of solid dispersion systems. J Pharm Sci 1971;60(09):1281-1302
14 Melocchi A, Loreti G, Del Curto MD, Maroni A, Gazzaniga A, Zema L. Evaluation of hot-melt extrusion and injection molding for continuous manufacturing of immediate-release tablets. J Pharm Sci 2015;104(06):1971-1980

15 Repka MA, Battu SK, Upadhye SB, et al. Pharmaceutical applications of hot-melt extrusion: Part II. Drug Dev Ind Pharm 2007;33 (10):1043-1057

16 Chmiel K, Knapik-Kowalczuk J, Jurkiewicz K, Sawicki W, Jachowicz $R$, Paluch $M$. A new method to identify physically stable concentration of amorphous solid dispersions (i): case of flutamide + kollidon VA64. Mol Pharm 2017;14(10):3370-3380

17 Hancock BC, Shamblin SL, Zografi G. Molecular mobility of amorphous pharmaceutical solids below their glass transition temperatures. Pharm Res 1995;12(06):799-806

18 Cross MM. Rheology of non-Newtonian fluids: a new flow equation for pseudoplastic systems. J Colloid Sci 1965;20(05):417-437

19 Cox WP, Merz EH. Correlation of dynamic and steady flow viscosities. J Polym Sci, Polym Phys Ed 1958;28(118):619-622

20 Yonemochi E, SanoS, Yoshihashi Y, et al. Diffusivity of amorphous drug in solid dispersion. J Therm Anal Calorim 2013;113(03):1505-1510

21 Farinas KC, Doh L, Venkatraman S, et al. Characterization of solute diffusion in a polymer using ATR-FTIR spectroscopy and bulk transport techniques. Macromolecules 1994;27(18):5220-5222

22 Xia J, Wang C. Holographic grating relaxation studies of probe diffusion in amorphous polymers. J Polym Sci, B, Polym Phys 1995;33(06):899-908

23 Kubicek M, Holzlechner G, Opitz AK, Larisegger S, Hutter H, Fleig J. A novel ToF-SIMS operation mode for sub $100 \mathrm{~nm}$ lateral resolution: application and performance. Appl Surf Sci 2014;289(100): 407-416

24 Mapes MK, Swallen SF, Kearns KL, Ediger MD. Isothermal desorption measurements of self-diffusion in supercooled o-terphenyl. J Chem Phys 2006;124(05):054710

25 Kothari K, Ragoonanan V, Suryanarayanan R. The role of polymer concentration on the molecular mobility and physical stability of nifedipine solid dispersions. Mol Pharm 2015;12(05):14771484

26 Yoshihashi Y, Lijima H, Yonemochi E, et al. Estimation of physical stability of amorphous solid dispersion using differential scanning calorimetry. J Therm Anal Calorim 2006;85(03):689-692

27 Aso Y, Yoshioka S. Molecular mobility of nifedipine-PVP and phenobarbital-PVP solid dispersions as measured by 13C-NMR spin-lattice relaxation time. J Pharm Sci 2006;95(02):318-325

28 Sun DD, Lee PI. Evolution of supersaturation of amorphous pharmaceuticals: nonlinear rate of supersaturation generation regulated by matrix diffusion. Mol Pharm 2015;12(04):1203-1215

29 Lee PI. Determination of diffusion coefficients by sorption from a constant, finite volume. In: Baker R, ed. Controlled Release of Bioactive Materials. 1st ed. New York, NY: Academic Press; 1980: 255-265

30 Sarode AL, Sandhu H, Shah N, Malick W, Zia H. Hot melt extrusion (HME) for amorphous solid dispersions: predictive tools for processing and impact of drug-polymer interactions on supersaturation. Eur J Pharm Sci 2013;48(03):371-384

31 Mapes MK, Swallen SF, Ediger MD. Self-diffusion of supercooled oterphenyl near the glass transition temperature. J Phys Chem B 2006;110(01):507-511

32 Ediger MD, Harrowell P, Yu L. Crystal growth kinetics exhibit a fragility-dependent decoupling from viscosity. J Chem Phys 2008; 128(03):034709

33 Treffer D, Troiss A, Khinast J. A novel tool to standardize rheology testing of molten polymers for pharmaceutical applications. Int J Pharm 2015;495(01):474-481

34 Alshahrani SM, Morott JT, Alshetaili AS, Tiwari RV, Majumdar S, Repka MA. Influence of degassing on hot-melt extrusion process. Eur J Pharm Sci 2015;80:43-52 
35 Menard KP, Bilyeu BW. Dynamic mechanical analysis of polymers and rubbers. In: Meyers RA, ed. Encyclopedia of Analytical Chemistry. Chichester: John Wiley \& Sons, Ltd; 2000:7562-7582

36 Chokshi RJ, Sandhu HK, Iyer RM, Shah NH, Malick AW, Zia H. Characterization of physico-mechanical properties of indomethacin and polymers to assess their suitability for hot-melt extrusion processs as a means to manufacture solid dispersion/solution. J Pharm Sci 2005;94(11):2463-2474

37 Crowley MM, Zhang F, Repka MA, et al. Pharmaceutical applications of hot-melt extrusion: part I. Drug Dev Ind Pharm 2007;33 (09):909-926

38 Cotts S, Potter C, DiPietro D. Materials Characterization by Thermal Analysis (DSC \& TGA), Rheology, and Dynamic Mechanical Analysis (Part 2). TA Instruments - Rheology and DMA Seminar September 2018. http://www.tainstruments.com/wp-content/ uploads/Materials_Characterization_Part2.pdf

39 DeBoyace K, Buckner IS, Gong Y, Ju TR, Wildfong PLD. Modeling and prediction of drug dispersability in polyvinylpyrrolidonevinyl acetate copolymer using a molecular descriptor. J Pharm Sci 2018;107(01):334-343

40 Avrami M. Kinetics of phase change. I: general theory.J Chem Phys 1939;7(12):1103-1112

41 Nurzyńska K, Booth J, Roberts CJ, McCabe J, Dryden I, Fischer PM. Long-term amorphous drug stability predictions using easily calculated, predicted, and measured parameters. Mol Pharm 2015;12(09):3389-3398

42 Bhardwaj SP, Arora KK, Kwong E, Templeton A, Clas SD, Suryanarayanan R. Correlation between molecular mobility and physical stability of amorphous itraconazole. Mol Pharm 2013;10 (02):694-700
43 Mehta M, Ragoonanan V, McKenna GB, Suryanarayanan R. Correlation between molecular mobility and physical stability in pharmaceutical glasses. Mol Pharm 2016;13(04):1267-1277

44 Ojo AT, Lee PI. Predicting the physical stability of amorphous solid dispersions: understanding the impact of material properties. Presented at: American Association of Pharmaceutical ScientistsPharmSci 360, San Antonio; 2019:Poster Number: M1530-04-28

45 Baghel S, Cathcart H, O'Reilly NJ. Polymeric amorphous solid dispersions: a review of amorphization, crystallization, stabilization, solid-state characterization, and aqueous solubilization of biopharmaceutical classification system class II drugs. J Pharm Sci 2016;105(09):2527-2544

46 Andraca A, Goldstein P, del Castillo LF. Tracer diffusion in glassforming liquids. Phys A Stat Mech 2008;387(18):4531-4540

47 Liu J, Cao D, Zhang L. Molecular dynamics study on nanoparticle diffusion in polymer melts: a test of the stokes-einstein law. J Phys Chem C 2008;112(17):6653-6661

48 Douglas J, Leporini D. Obstruction model of the fractional StokesEinstein relation in glass-forming liquids. J Non-Cryst Solids 1998;235-237:137-141

49 Puosi F, Pasturel A, Jakse N, Leporini D. Communication: fast dynamics perspective on the breakdown of the Stokes-Einstein law in fragile glassformers. J Chem Phys 2018;148(13):131102

50 Deppe DD, Dhinojwala A, Torkelson JM. Small molecule probe diffusion in thin polymer films near the glass transition: a novel approach using fluorescence nonradiative energy transfer. Macromolecules 2002;29(11):3898-3908

51 Mason TG. Estimating the viscoelastic moduli of complex fluids using the generalized Stokes-Einstein equation. Rheol Acta 2000; 39(04):371-378 